\title{
DYNAMIC STRUCTURES FOR NON-UNIFORM SUBBAND ADAPTIVE FILTERING
}

\author{
Amere Oakman and Patrick Naylor \\ CSP Group, Dept. of Electrical and Electronic Engineering \\ Imperial College of Science, Technology and Medicine \\ London, UK
}

\begin{abstract}
Subband adaptive filters suffer degraded performance when high input energy occurs at frequencies coincident with subband boundaries. This is seen as increased error in critically sampled systems and as reduced asymptotic convergence speed in oversampled systems. To address this problem a dynamic frequency decomposition scheme is presented which aims to control the frequency of subband boundaries such that they avoid spectral regions of high input energy. An efficient structure for this is described, which maintains the low complexity advantage of subband systems. Simulation results show reductions in MSE of around $5-10 \mathrm{dBs}$ in the critical case and convergence improvement in the oversampled case, in addition to increased robustness to coloured inputs in both cases.
\end{abstract}

\section{INTRODUCTION}

Subband adaptive filters (SAF) are used in system identification applications such as acoustic echo cancellation where the unknown system can be of the order of several thousand taps. They have the main benefits of reduced complexity and possible increased convergence speed due to reduction of eigenvalue spread in the subband signals [1]. Errors in both critically and oversampled SAFs can be shown to be related to signal components around subband boundaries. In the case of critical sampling this manifests itself as dominating peaks in the final error signal around the subband boundaries [2]. In the case of oversampling, slow asymptotic convergence is observed [3].

Our approach is to use non-uniform dynamic frequency-subband decomposition (NDS) to substantially reduce these errors. The algorithm chooses the decomposition so as to avoid high-energy signal components around subband boundaries, whilst retaining high decimation factors when possible so as to keep complexity low.

Previous work in [4] [5] has been extended in this paper by introducing a filterbank structure appropriate for efficient NDS and examining the exact sources of error in uniform SAFs. These are crucial issues, as no suitable dynamic structure has been previously suggested and if complexity is not taken into account, improvements in performance cannot be evaluated consistently. Secondly, knowledge of the sources of error is essential for correct choice of decomposition. The following sections present a general filterbank structure that will allow the dynamic alteration of subband frequency decomposition, and a control algorithm for the dynamic structure that reduces errors in SAFs around subband boundaries. Simulations are presented for both the critically and oversampled cases to demonstrate the effectiveness of the scheme.

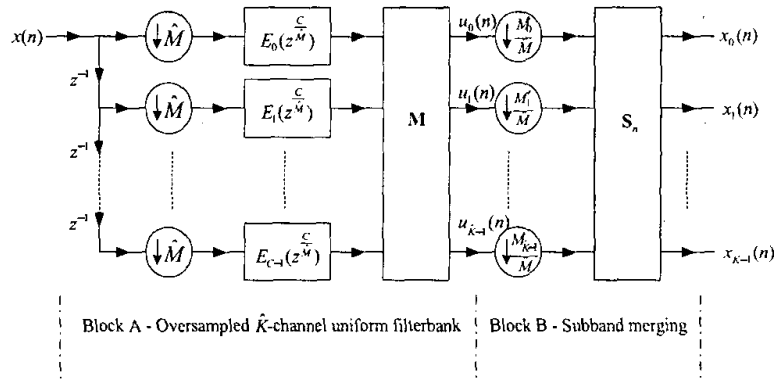

Fig. 1. General structure for dynamic filterbank

\section{DYNAMIC FILTERBANK STRUCTURE}

In our approach a non-uniform filterbank (NUFB) is obtained by merging the subbands of a $\widehat{K}$-channel uniform filterbank (UFB) and then decimating each of the resulting $K(\leq \widehat{K})$ subbands by an integer factor $M_{k}$ [6]. This structure is modified to provide an efficient method of NDS. The structure has the general form of Figure 1 , where $x(n)$ is the fullband input signal, $E_{c}(z)$ are polyphase components of a prototype lowpass filter $P(z), \mathbf{M}$ is a modulation matrix, $\mathbf{S}_{n}$ is a time-varying summation matrix consisting of ones and zeros, and $x_{k}(n)$ are the $k$ subband signals. The first stage of decimation is by a constant integer, $\widehat{M}$, whilst the second stage is by a time-varying integer, $M_{k, n} / \widehat{M}$, for the $k^{\text {th }}$ subband. Strictly, $k$ should be denoted $k_{n}$ since it is timevarying, however this is usually dropped for clarity. $\widehat{M}$ represents the lowest decimation factor in the structure and therefore defines the largest subband channel bandwidth, decided by the application and as the greatest common denominator of all possible $M_{k, n}$

$$
\widehat{M}=\operatorname{gcd}\left(M_{k, n}\right) \forall k, n
$$

to provide maximum decimation.

In Figure 1 Block $A$ is an oversampled UFB and Block B is a subband merging section. This structure is preferred since $A$ can be implemented efficiently using fast transforms of the outputs of a decimated polyphase network [7] and B consists solely of adders and decimators. The structure also allows NDS by changes in B only, without the need for intermediate upsampling/downsampling. The synthesis bank will be the mirror of Figure l.

Disregarding for the moment important implementation details to be discussed, which additionally specify Block $\mathrm{A}$, the struc- 
ture can be viewed as a generalisation, via the Noble identities [7], of the static structures in [6][8]. Near-perfect reconstruction is achieved for NUFBs derived from UFBs in this way, iff the following condition is met [8]

$$
l_{k}=\alpha_{k} n_{k}, \quad k=0,1, \ldots, K-1
$$

where the summation of $l_{k}$ bandpass filters, whose passbands extend from $n_{k} \pi / \widehat{K}$ to $\left(n_{k}+l_{k}\right) \pi / \widehat{K}$, form the $k^{\text {th }}$ analysis filter and $\alpha_{k}$ is any positive integer.

\subsection{Critically sampled implementation}

Block A is an oversampled, uniform, modulated filterbank. In the case of a critically sampled NUFB, Block A can be implemented in a standard way, for example as a cosine-modulated filterbank (CMF) by setting $C=2 \widehat{K}$ and $\mathbf{M}$ to be a $(\widehat{K} \times \widehat{K})$ cosine modulation matrix combined with a complex-conjugate summation matrix [7]. This will produce the desired subbands as long as $\widehat{M}$ satifies (1). Implementing the modulation using a fast DCT [9] gives an overall computational complexity for Block $\mathrm{A}$, in terms of real multiplies per fullband sample period ( $\mathrm{rmfp}$ ),

$$
(1 / \widehat{M})\left(L_{p}+\left((\widehat{K} / 2) \log _{2} \widehat{K}+\widehat{K}\right)\right)
$$

where $L_{p}$ is the length of the prototype filter $P(z)$. Since for this structure $\widehat{M} \leq \widehat{K}$, the computational cost of the filterbank is slightly greater then for a classical non-dynamic filterbank. However, this computational penalty can be compensated for by reducing the subband complexity whilst maintaining significant performance advantages over equivalent complexity static structures, as shall be seen.

\subsection{Oversampled implementation}

In the oversampled case the implementation of Block A is less straightforward. Let $N_{k}=\widehat{K} / M_{k}$ be the oversampling ratio for the $k^{\text {th }}$ subband. If $N_{k}$ is an integer, $C=\widehat{K}$ or $C=2 \widehat{K}$ (for a DFT filterbank or a CMF respectively) and $\widehat{M}$ satisfies (1), then the polyphase components $E_{c}\left(z^{C / \widehat{M}}\right)$ are expressed in terms of integer powers of $z$. If $N_{k}$ is not an integer, then $C / \widehat{M}$ cannot always be an integer unless $\widehat{M}=1$, in which case all Block A operations will be carried out at the fullband rate, even if $N_{k} \simeq 1$, otherwise fractional delays are required.

There are several options for efficient use of fractional oversampling ratios [10]. Our preferred implementation is where all subbands are complex-valued and arranged such that processing of only half the number of subbands is necessary (the others being complex-conjugates), allowing $N_{k} \simeq 1$ and making the overall computational complexity almost equivalent to real-subband processing. To achieve this, $C$ is the least common multiple of $(\widehat{M}, \widehat{K})$ and $\mathbf{M} \equiv \mathbf{W}_{C}\left(e^{j \omega}\right) \equiv(C \mathrm{x} C)$ DFT matrix (which includes redundant terms), ensuring that the delays in the $E_{c}(z)$ terms are integer-valued and that the polyphase outputs are correctly modulated. Further factorisation of $\mathbf{M}$ enables $\mathbf{W}_{C}\left(e^{j \omega}\right)$ to be implemented by a $\widehat{K}$-length FFT giving a computational complexity (rmfp) [10],

$$
(1 / \widehat{M})\left(L_{p}+\left(2 \widehat{K} \log _{2} \widehat{K}+2 \widehat{K}\right)\right)
$$

Importantly, if the prototype filter $P(z)$ is designed to have the stopband beginning at $\pi / \max \left(M_{k}\right)$ then the condition (2) can be ignored, since aliasing is limited to the stopband attenuation of $P(z)$. This allows more flexibility in the choice of decomposition.

\section{CONTROL ALGORITHM}

The use of NDS schemes for SAF is suggested for two cases below. Both using the same basic approach: $K_{0}=\widehat{K}$, i.e. all subbands have minimum bandwidth at $n=0$, giving the greatest resolution for merging decisions. The adaptation then proceeds in a blockwise fashion, in which merging decisions are made at the end of each block based on the criterion for the two cases. Smaller bandwidth subbands are retained where possible, as they provide the highest efficiency. The subband complexity (rmpf) for the NLMS algorithm is

$$
C_{\text {sub }}=2 \sum_{k=0}^{K-1}\left(L_{\text {total }} / M_{k}^{2}\right)
$$

where $L_{\text {total }}$ is the total number of taps in the structure. Subband merging is done binary-tree-wise, with two adjacent subbands being merged at a time. This satisfies (2) and provides an efficient implementation - however the structure or criterion given are not limited to this merging scheme.

\subsection{Critically sampled case}

Inband aliasing degrades the performance of critically sampled SAFs around the subband boundaries [2]. Consider, without loss of generality, a 2-band QMF SAF system with prototype $P(z)$, unknown system $S(z)$ and input $X(z)$. The fullband error of this system is given by [11]

$$
\begin{aligned}
E(z)= & P(z) P(-z) . \\
& {\left[\begin{array}{c}
P(z) P(-z)(S(z)-S(-z)) \\
\left(P^{2}(z)-P^{2}(-z)\right)(S(z)-S(-z))
\end{array}\right]^{\mathrm{T}} \mathbf{X}(z) }
\end{aligned}
$$

where $\mathbf{X}(z)=[X(z) X(-z)]$. The error mainly comprises aliased input at the subband boundaries [2]. The motivation for NDS is to remove the subband boundaries which contribute most to $E(z)$, thereby reducing the overall error. Hence in the $b^{\text {th }}$ (length $B$ ) block of samples the mean square input power for each subband $\bar{x}_{k, b}$ is calculated as

$$
\bar{x}_{k, b}=(1 / B) \sum_{m=n B}^{n(B+1)-1} x_{k}^{2}(m)
$$

which is assumed to be proportional to the error it induces in (6) and hence adjacent subbands are merged if $\bar{x}_{k, b}$ is higher than the mean over all subbands. It cannot be guaranteed that simply reducing the number of subbands uniformly or using a different static decomposition would reduce these boundary effects, since they are dependent upon $X(z)$ and to a lesser extent $S(z)$.

\subsection{Oversampled case}

In the oversampled case inband aliasing is limited to the stopband attenuation of the filterbank. This is typically large enough such that the error source identified in (6) is practically eliminated. In this case, the use of NDS addresses the slow asymptotic convergence [3]. The MSE $\xi_{k, n}$ in the $k^{\text {th }}$ subband, at time $n$, can be expressed as [3]

$$
\xi_{k, n}=\xi_{k, \min }+\sum_{l=0}^{L-1}\left|\phi_{k, l}^{\mathrm{H}} \mathbf{c}_{k}\right|^{2} \lambda_{k, l}\left(1-\mu_{k} \lambda_{k, l}\right)^{2 n}
$$



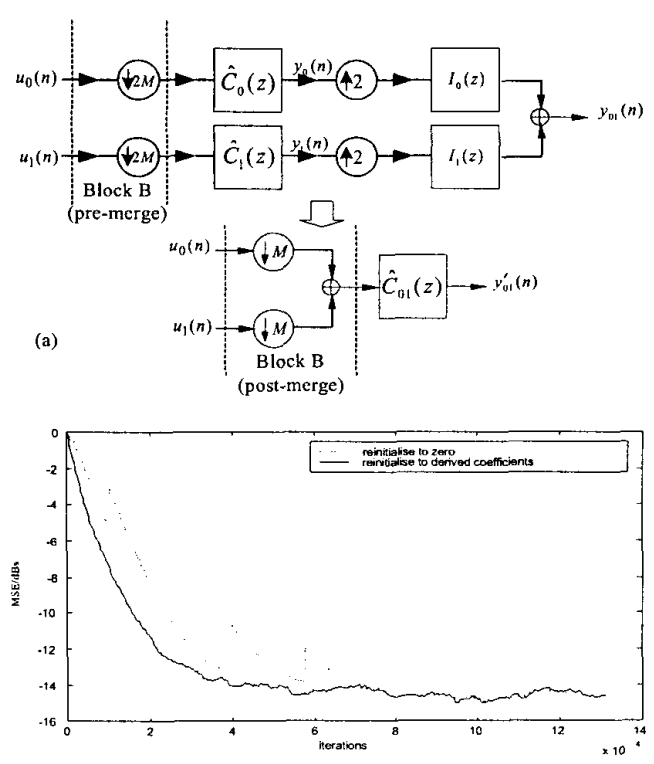

(b)

Fig. 2. (a) Subband merging (b) simulation showing result for two different reinitialisation schemes

where $\mathbf{c}_{k}$ is the length $L$ Wiener-Hopf solution in the $k^{\text {th }}$ subband and $\phi_{k, l}^{\mathrm{H}}$ and $\lambda_{k, l}$ are the eigenvectors and eigenvalues, respectively, of the $(L \mathrm{x} L)$ subband autocorrelation matrix $\mathbf{R}_{k}=\mathrm{E}\left\{\mathbf{x}_{k} \mathbf{x}_{k}^{\mathrm{T}}\right\}$, where $\mathbf{x}_{k}$ is the $k^{\text {th }}$ subband input. It has been shown that the most significant terms of (8) are due to energy around the subband edges [3]. The objective then is to remove boundaries where the subband input or subband desired signal is large, increasing the convergence rate. Hence the mean-squared-value of the subband input $\bar{x}_{k, b}$ and the subband desired signals $\bar{d}_{k, b}$ are calculated as in (7) and adjacent subbands are merged if $\bar{x}_{k, b}+\bar{d}_{k, b}$ is larger than the mean-value over all subbands.

\section{SUBBAND MERGING}

In this section we address the details of subband merging of adaptive filters. This may be viewed as in Figure 2(a) for the binarytree merging mentioned previously, where $\widehat{C}_{01}(z)$ results from the merging of subband filters $\widehat{C}_{0}(z)$ and $\widehat{C}_{1}(z), I_{0,1}(z)$ are ideal low and highpass filters respectively with cutoff at $\pi / 2$, and $u_{0,1}(n)$ are subband signals from Figure $1 . Y_{01}(z)$ and $Y_{01}^{\prime}(z)$ are given by (ignoring aliasing terms due to decimation since $I_{0,1}(z)$ are ideal)

$$
\begin{gathered}
Y_{01}(z)=I_{0}(z) \widehat{C}_{0}\left(z^{2}\right) U_{0}\left(z^{\frac{1}{M}}\right)+I_{1}(z) \widehat{C}_{1}\left(z^{2}\right) U_{1}\left(z^{\frac{1}{M I}}\right) \\
Y_{01}^{\prime}(z)=\widehat{C}_{01}(z) U_{0}\left(z^{\frac{1}{M}}\right)+\widehat{C}_{01}(z) U_{1}\left(z^{\frac{1}{M}}\right)
\end{gathered}
$$

If we equate (9) and (10), using $I_{1}(z)=I_{0}(-z)$, then $\widehat{C}_{01}(z)$ is

$$
\widehat{C}_{01}(z)=I_{0}(z) \widehat{C}_{0}\left(z^{2}\right)+I_{0}(-z) \widehat{C}_{1}\left(z^{2}\right)
$$

which is truncated to the length of the new subband adaptive filter. In practice $I_{0,1}(z)$ are non-ideal and hence an approximation of $\widehat{C}_{01}(z)$ is used, however no significant degradations are observed.

If the subbands are formed through purely real operations (e.g. the CMF) band-position must be taken into account, as this will determine whether the subband signal contains the positive or negative frequency spectrum part of the input. When $\widehat{C}_{01}(z)$ differs in this with respect to $\widehat{C}_{0}(z), \widehat{C}_{1}(z)$, it must be shifted by $\pi$. This can be seen by considering the critical decimation of a real bandpass signal. The reinitialisation of merged subband filters in this case can be written as

$\widehat{C}_{k, k \pm 1}\left(e^{j \omega}\right)=\left\{\begin{array}{l}\widehat{C}_{k, k \pm 1}^{\prime}\left(e^{j \omega}\right),\left(\frac{1}{\widehat{K}} \sum_{i=0}^{\max (k, k \pm 1)} l_{i}\right)_{\text {mud } 2}=0 \\ \widehat{C}_{k, k \pm 1}^{\prime}\left(e^{j(\omega+\pi)}\right),\end{array}\right.$

where $\widehat{C}_{k, k \pm 1}^{\prime}\left(e^{j \omega}\right)=I_{0}( \pm z) \widehat{C}_{k}\left(z^{2}\right)+I_{1}( \pm z) \widehat{C}_{k \pm 1}\left(z^{2}\right), \widehat{K}$ is the number of constituent subbands and $l_{i}$ is as in (2). Conditions for other merging schemes are derived from similar consideration of critical decimation of real bandpass signals. Figure 2(b) shows an example of reinitialisation of SAF taps after merging using this scheme (solid line) compared with a simple reinitialisation to zero (dashed line), which causes transients in the error

\section{SIMULATIONS}

\subsection{Critical case}

To test the performance of NDS a coloured input $x(n)$ has been chosen, generated by filtering a Gaussian white noise (GWN) process with $F(z)=\frac{0.5+0.34 z^{-1}+0.4 z^{-2}}{1+0.76 z^{-1}+0.981 z^{-2}}$ (truncated to 1024 taps), and the unknown system is $S(z)=z^{-1023}$. This is an insightful case since the input spectrum has a peak which, in certain cases, falls at a subband boundary and $S(z)$ is realistic in length and has a flat spectrum ensuring performance differences are due to $x(n)$ only. Five NDS systems with $\widehat{K}=16$ and $\widehat{M}=4$ are compared with five uniform SAFs with $K=8,7,6,5,4$, where the maximum overall complexity (including the filterbanks) of the NDS systems is set to the complexity of each of the uniform systems. Figure 3(a) shows the results from the uniform SAFs to be highly variable. For $K=8$, there is little convergence because of the spectral peak in the input exactly at a subband boundary. The $K=4$ system performs best, as it has the peak exactly in the centre of one of its subbands. The systems $K=7,6,5$ perform at various levels and computational complexity does not govern performance, as we have increasing error for $K=7,6,5$. Figure 3 (b), shows that the performance of each NDS system is approximately equal and that the error increases with decreasing complexity ( $K$ increasing). The dynamic frequency decomposition can be seen to be converging during the first 2000 iterations to solutions that avoid placing a subband boundary in the spectral region with high input energy.

\subsection{Oversampled case}

To demonstrate the MSE behaviour in (8), $x(n)$ is chosen to be GWN and the unknown system to be $F(z)$. Five NDS systems have $\widehat{K}=32$ bands (only $\widehat{K} / 2$ are processed) and minimum oversampling ratio $N_{N D S}=8 / 7$. These are compared against 5 uniform systems of increasing complexity, with $K=16,14,12,10,8$, 
(a)

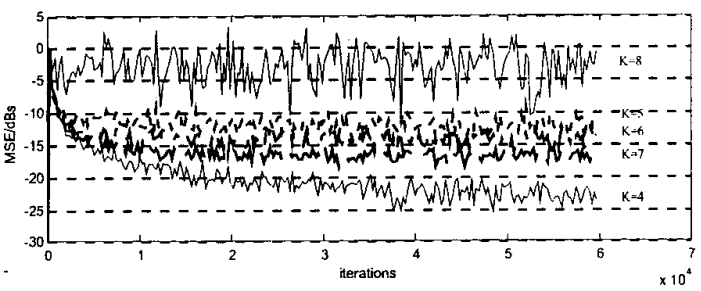

(b)

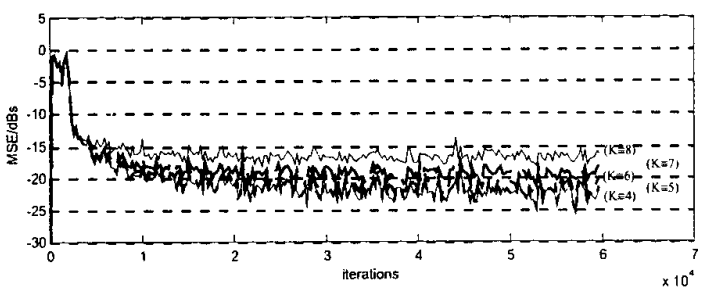

Fig. 3. Uniform and adaptive critical SAF systems - (a) uniform fixed decomposition (b) NDS with fixed maximum complexity

with oversampling ratios $N_{U} \simeq 8 / 7$. Again the maximum overall complexity of the NDS systems was set to that of the corresponding uniform system. Figure 4(a), shows a variation of convergence speeds and no consistent relation between complexity and performance, for the uniform fixed systems. In Figure 4(b), there is significantly less variation and no situation when an increase in complexity results in a decrease in performance, for the NDS systems.

\section{CONCLUSIONS}

This paper has presented a dynamic non-uniform filterbank structure that reduces performance limitations of static subband adaptive systems with coloured inputs. Analysis of the complexity of the structures and of merging of subband adaptive filters is given. Simulations show that the robustness of NDS to highly coloured inputs yields improvements of around 5-10dBs in MSE in the critical case without increasing complexity. In the oversampled case, corresponding improvements in convergence speed are particularly significant. The dynamic system overcomes the problems of static systems by finding a subband decomposition that avoids subband boundaries in spectral regions with high input power and/or high unknown system gain. The oversampled structure is preferred since, in the critical case, the constraints on subband decomposition limit flexibility.

\section{REFERENCES}

[1] W. Kellerman, "Analysis and design of multirate systems for the cancellation of acoustical echoes," in Proc. IEEE Int. Conf. On Acoustics, Speech and Signal Processing, New York, NY, April, 1988, pp. 2570-2573.

[2] A. Gilloire and M. Vetterli, "Adaptive filtering in subbands with critical sampling: Analysis, experiments, and application to acoustic echo cancellation," IEEE Transactions on Signal Processing, vol. 40, no. 8, pp. 1862-1875, 1992. (a)

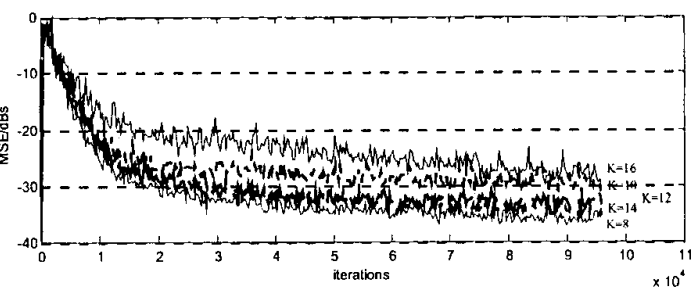

(b)

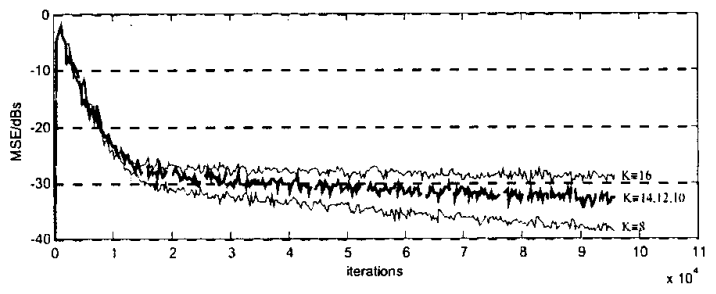

Fig. 4. Uniform and adaptive oversampled SAF systems - (a) uniform fixed decomposition (b) NDS with fixed maximum complexity

[3] D.R. Morgan, "Slow asymptotic convergence of LMS acoustic echo cancellers," IEEE Transactions on Speech and Audio Processing, vol. 3, no. 2, pp. 126-136, Mar. 1995.

[4] M.L. McCloud and D.M. Etter, "A technique for nonuniform subband adaptive filtering with varying bandwidth filter banks," in Conference Record of the Thirtieth Asilomar Conference on Signals, Systems and Computers, 1996, 1997, vol. 2, pp. 1315-1318.

[5] J.D. Griesbach, T. Bose, and D.M. Etter, "Non-uniform filterbank bandwidth allocation for system modelling subband adaptive filters," in Proc. IEEE Int. Conf. On Acoustics, Speech and Signal Processing, 1999, vol. 3, pp. 1473-1476.

[6] Jeong-Jin Lee and Byeong Gi Lee, "A design of nonuniform cosine modulated filter banks," IEEE Transactions on Circuits and Systems-II:Analog and Digital Signal Processing, vol. 42, pp. 732-737, 1995.

[7] P.P. Vaidyanathan, Multirate Systems and Filler Banks, Prentice Hall, englewood cliffs, NJ, 1993.

[8] J. Li, T.Q. Nguyen, and S. Tantaratana, "A simple design method for near-perfect-reconstruction nonuniform filter banks," IEEE Trans. On Signal Processing, vol. 45, no. 8, pp. 2105-2109, Aug. 1997.

[9] P. Yip and K.R. Rao, "Fast decimation-in-time algorithms for a family of discrete cosine and sine-transforms," Circuits, Systems and Signal Processing, vol. 3, no. 4, pp. 387-408, 1984.

[10] Z. Cvetkovic and M. Vetterli, "Oversampled filter banks," IEEE Trans. On Signal Processing, vol. 46, no. 5, pp. 12451255, May 1998.

[11] A. Gilloire and M. Vetterli, "Adaptive filtering in sub-bands," in Int. Conf. On Acoustics, Speech and Signal Processing, Apr. 1988, vol. 3, pp. 1572-1575. 